\title{
DARBOUX TRANSFORMATION \\ FOR THE SCHRÖDINGER EQUATION \\ WITH STEPLIKE POTENTIALS
}

\author{
Tuncay Aktosun \\ Department of Mathematics \\ North Dakota State University \\ Fargo, ND 58105
}

\begin{abstract}
The one-dimensional Schrödinger equation is considered when the potential is asymptotic to a positive constant on the right half line. The corresponding Darboux transformation is established by showing how the scattering solutions, the scattering coefficients, and the potential change when bound states are added or removed. The scattering coefficients are represented as certain integrals, from which their properties can be directly extracted.
\end{abstract}

PACS Numbers: 03.65.Nk, 03.80.+r

Mathematics Subject Classification (1991): 34L25, 34L40, 81U05

Keywords: 1-D Schrödinger equation, Nondecaying potentials, Steplike potentials, Darboux transformation, Bound states

Short title: Darboux transformation for steplike potentials 


\section{INTRODUCTION}

Consider the one-dimensional Schrödinger equation

$$
\psi^{\prime \prime}(k, x)+k^{2} \psi(k, x)=V(x) \psi(k, x), \quad x \in \mathbf{R},
$$

where the potential $V$ is real valued and satisfies

$$
V \in L_{1}^{1}\left(\mathbf{R}^{-}\right), \quad V-c^{2} \in L_{1}^{1}\left(\mathbf{R}^{+}\right),
$$

for some $c \geq 0$. In our notation, the prime denotes the derivative with respect to the spatial variable $x, \mathbf{R}^{-}=(-\infty, 0), \mathbf{R}^{+}=(0,+\infty)$, and $L_{1}^{1}$ is the set of measurable functions $f$ on an interval $I$ such that $\int_{I} d x(1+|x|)|f(x)|$ is finite. We will use $\mathbf{C}^{+}$to denote the upper half complex plane and $\overline{\mathbf{C}^{+}}=\mathbf{C}^{+} \cup \mathbf{R}$.

Our main goal is to analyze the Darboux transformation for (1.1), namely, to understand how the scattering solutions, the scattering coefficients, and the potential change when bound states are added or removed. The Darboux transformation when $c=0$ in (1.2) is well understood [1,2]. For a more general treatement of Darboux transformations, the reader is referred to [3] and the references therein. In the limit $c \rightarrow 0$, the transformation we present in Section 4 reduces to the well-known case. The main difficulty when $c>0$ is the analysis at $k \in \mathbf{C}^{+}$as $x \rightarrow+\infty$ of the behavior of $f_{r}(k, x)$, the Jost solution from the right defined in Section 2. We overcome this difficulty by working with a regular solution of (1.1) analyzed in Section 3.

The bound states of (1.1) are its square-integrable solutions, whereas the scattering states of (1.1) correspond to solutions behaving like $e^{ \pm i k x}$ as $x \rightarrow-\infty$ and like $e^{ \pm i \gamma x}$ as $x \rightarrow+\infty$, where

$$
\gamma=\sqrt{k^{2}-c^{2}},
$$

in which the branch of the square-root function is used with $\operatorname{Im} \gamma \geq 0$. Thus, $\gamma$ is purely imaginary when $k \in(-c, c)$. 
The reader is referred to [4-7] for the analysis of the direct and inverse scattering

problems for (1.1). For a more general analysis of the scattering problem, see also $[8,9]$ and the references therein. The inverse scattering problem for (1.1), namely the recovery of $V$ from an appropriate set of scattering data, has important applications [10-12] in the recovery of material properties of thin films. Thus, we expect our results to be useful in $\mathrm{X}$-ray and neutron reflectometry [11-15].

Our paper is organized as follows. In Section 2 we review some relevant properties of the scattering solutions and the bound states. In Section 3, we obtain various properties of a regular solution of (1.1) that are needed in establishing the Darboux transformation. In Section 4 we present the Darboux transformation and show how the bound states can be added or removed. Finally, in Section 5 we evaluate the spatial asymptotics of Jost solutions and present some integral representations of the scattering coefficients.

\section{JOST SOLUTIONS AND SCATTERING COEFFICIENTS}

Among the scattering solutions of (1.1) are the so-called Jost solutions with specific boundary conditions at $x= \pm \infty$. The Jost solution from the left, $f_{l}(k, x)$, associated with $V$ is the solution of (1.1) satisfying

$$
e^{-i \gamma x} f_{l}(k, x)=1+o(1), \quad e^{-i \gamma x} f_{l}^{\prime}(k, x)=i \gamma+o(1), \quad x \rightarrow+\infty,
$$

where $\gamma$ is the quantity defined in (1.3). It satisfies the integral relation

$$
f_{l}(k, x)=e^{i \gamma x}+\frac{1}{\gamma} \int_{x}^{\infty} d y \sin \gamma(y-x)\left[V(y)-c^{2}\right] f_{l}(k, y) .
$$

Similarly, $f_{r}(k, x)$, the Jost solution from the right, is defined as the solution of (1.1) satisfying

$$
e^{i k x} f_{r}(k, x)=1+o(1), \quad e^{i k x} f_{r}^{\prime}(k, x)=-i k+o(1), \quad x \rightarrow-\infty,
$$


and it satisfies the integral relation

$$
f_{r}(k, x)=e^{-i k x}+\frac{1}{k} \int_{-\infty}^{x} d y \sin k(x-y) V(y) f_{r}(k, y)
$$

We later need the following known properties [4,5] of the Jost solutions.

Proposition 2.1 Assume $V$ satisfies (1.2) for some $c \geq 0$. Then, for each fixed $x \in \mathbf{R}$, the functions $f_{l}(k, x), f_{l}^{\prime}(k, x), f_{r}(k, x)$, and $f_{r}^{\prime}(k, x)$ are analytic in $k \in \mathbf{C}^{+}$and continuous in $k \in \overline{\mathbf{C}^{+}}$. Moreover, for each fixed $k \in \overline{\mathbf{C}^{+}}$, these four functions are continuous in $x \in \mathbf{R}$.

The transmission and reflection coefficients from the left, $T_{l}$ and $L$, can be defined in terms of the spatial asymptotics of $f_{l}$ as

$$
e^{-i k x} f_{l}(k, x)=\frac{1}{T_{l}(k)}+\frac{L(k)}{T_{l}(k)} e^{-2 i k x}+o(1), \quad x \rightarrow-\infty, \quad k \in \mathbf{R} \backslash\{0\}
$$

Similarly, the transmission and reflection coefficients from the right, $T_{r}$ and $R$, can be defined in terms of the spatial asymptotics of $f_{r}$ as

$$
e^{i \gamma x} f_{r}(k, x)=\frac{1}{T_{r}(k)}+\frac{R(k)}{T_{r}(k)} e^{2 i \gamma x}+o(1), \quad x \rightarrow+\infty, \quad \gamma \in \mathbf{R} \backslash\{0\}
$$

Since (2.6) holds only for $k \in \mathbf{R} \backslash[-c, c]$, one needs to use other means to define $R(k)$ and $T_{r}(k)$ for $k \in[-c, c]$. It turns out $[4,5]$ that

$$
\begin{aligned}
T_{r}(k) & =\frac{\gamma}{k} T_{l}(k), \quad k \in \overline{\mathbf{C}^{+}} \backslash\{0\}, \\
R(k) & =-\frac{L(k)^{*} T_{l}(k)}{T_{l}(k)^{*}}, \quad k \in \mathbf{R},
\end{aligned}
$$

where the asterisk denotes complex conjugation. The reader is referred to $[4,5,7]$ for the small- $k$ asymptotics of the scattering coefficients. The poles of $T_{l}$ in $\mathbf{C}^{+}$correspond $[4,5]$ to the bound states of (1.1). Under (1.2) it is known $[4,5,7]$ that such poles are simple, confined to the positive imaginary axis, and finite in number. Let us assume that there are $N$ bound states at $k=i \kappa_{j}$ with $0<\kappa_{1}<\cdots<\kappa_{N}$. 
Let $[f ; g]=f g^{\prime}-f^{\prime} g$ denote the Wronskian. It is well known that the Wronskian of any two solutions of (1.1) are independent of $x$. From (2.3) and (2.5) it follows that

$$
\frac{1}{T_{l}(k)}=\frac{1}{2 i k}\left[f_{r}(k, x) ; f_{l}(k, x)\right],
$$

and hence $f_{l}(k, x)$ and $f_{r}(k, x)$ are linearly dependent at the bound states and linearly independent otherwise. In fact, $f_{l}\left(i \kappa_{j}, x\right)$ and $f_{r}\left(i \kappa_{j}, x\right)$ decay exponentially $[4,5]$ to zero as $x \rightarrow \pm \infty$. Thus, if we let

$$
\mu_{j}:=\frac{f_{l}\left(i \kappa_{j}, x\right)}{f_{r}\left(i \kappa_{j}, x\right)},
$$

then each $\mu_{j}$ is independent of $x$ and is a real nonzero constant.

Proposition 2.2 Assume $V$ satisfies (1.2) for some $c \geq 0$ with the bound states occurring at $k=i \kappa_{j}$ for $j=1, \ldots, N$. Then, both $f_{l}(i \kappa, x)$ and $f_{r}(i \kappa, x)$ are strictly positive when $\kappa \geq \kappa_{N}$. In case there are no bound states, $f_{l}(i \kappa, x)$ and $f_{r}(i \kappa, x)$ are strictly positive for all $\kappa>0$.

PROOF: The proof is similar to the case when $c=0$ and it can be obtained, e.g., by using Proposition 10.1 of [16].

Proposition 2.3 Assume $V$ satisfies (1.2) for some $c \geq 0$ with the bound states occurring at $k=i \kappa_{j}$ for $j=1, \ldots, N$. Then:

(i) $T_{l}(i \kappa)>0$ when $\kappa>\kappa_{N}$.

(ii) $(-1)^{j} T_{l}(i \kappa)>0$ when $\kappa \in\left(\kappa_{N-j}, \kappa_{N-j+1}\right)$ for $j=1, \ldots, N-1$.

(iii) $(-1)^{N} T_{l}(i \kappa)>0$ when $\kappa \in\left(0, \kappa_{1}\right)$.

If there are no bound states, then $T_{l}(i \kappa)>0$ for $\kappa>0$.

PROOF: The proof is obtained by noticing $[4,5]$ that $1 / T_{l}(i \kappa)$ is real and continuous for 
$\kappa \in \mathbf{R}$, it has simple zeros at $\kappa=\kappa_{j}$ for $j=1, \ldots, N$, and that it converges to 1 as $\kappa \rightarrow+\infty$

\section{REGULAR SOLUTION}

Let $v(k, x)$ be the solution of (1.1) satisfying the boundary conditions

$$
v(k, 0)=0, \quad v^{\prime}(k, 0)=1 .
$$

For each fixed $x \in \mathbf{R}, v(\cdot, x)$ is entire on the complex plane and hence it is a 'regular' solution. As in (3.3) and (3.5) of [7] we have the integral relations

$$
\begin{aligned}
& v(k, x)= \begin{cases}\frac{\sin \gamma x}{\gamma}+\frac{1}{\gamma} \int_{0}^{x} d y \sin \gamma(x-y)\left[V(y)-c^{2}\right] v(k, y), \quad x \geq 0 \\
\frac{\sin k x}{k}+\frac{1}{k} \int_{x}^{0} d y \sin k(y-x) V(y) v(k, y), & x \leq 0,\end{cases} \\
& v^{\prime}(k, x)= \begin{cases}\cos \gamma x+\int_{0}^{x} d y \cos \gamma(x-y)\left[V(y)-c^{2}\right] v(k, y), & x \geq 0, \\
\cos k x-\int_{x}^{0} d y \cos k(y-x) V(y) v(k, y), & x \leq 0 .\end{cases}
\end{aligned}
$$

From (3.1) and the constancy of the Wronskian of any two solutions of (1.1), it follows that

$$
\left[f_{l}(k, x) ; v(k, x)\right]=f_{l}(k, 0), \quad\left[f_{r}(k, x) ; v(k, x)\right]=f_{r}(k, 0) .
$$

Let us fix $\kappa>\kappa_{N}(\kappa>0$ if (1.1) has no bound states). When a bound state is added to (1.1) at $k=i \kappa$, we are interested in finding the potential, the scattering coefficients, and the Jost solutions corresponding to the resulting Schrödinger equation. For this, we prove several propositions that are needed to establish for the Darboux transformation formulas in Section 4. 
From (2.9), (3.4), and Propositions 2.2 and 2.3, it follows that any two of $f_{l}(i \kappa, x)$, $f_{r}(i \kappa, x)$, and $v(i \kappa, x)$ are linearly independent. Thus, we have

$$
\begin{array}{ll}
f_{r}(i \kappa, x)=A_{1}(\kappa) f_{l}(i \kappa, x)+A_{2}(\kappa) v(i \kappa, x), & x \geq 0, \\
f_{l}(i \kappa, x)=A_{3}(\kappa) f_{r}(i \kappa, x)-A_{4}(\kappa) v(i \kappa, x), & x \leq 0,
\end{array}
$$

where the coefficients $A_{j}(\kappa)$ are analyzed in the next proposition.

Proposition 3.1 Assume $V$ satisfies (1.2) for some $c \geq 0$ and that $\kappa>\kappa_{N}$ (if there are no bound states, let $\kappa>0)$. Then, all the four $A_{j}(\kappa)$ appearing in (3.5) and (3.6) are strictly positive.

PROOF: Using (2.9) and (3.4)-(3.6) we get

$$
\begin{gathered}
A_{1}(\kappa)=\frac{1}{A_{3}(\kappa)}=\frac{f_{r}(i \kappa, 0)}{f_{l}(i \kappa, 0)} \\
A_{2}(\kappa)=\frac{2 \kappa}{T_{l}(i \kappa) f_{l}(i \kappa, 0)}, \quad A_{4}(\kappa)=\frac{2 \kappa}{T_{l}(i \kappa) f_{r}(i \kappa, 0)} .
\end{gathered}
$$

By Propositions 2.2 and 2.3 all the three quantities $f_{l}(i \kappa, 0), f_{r}(i \kappa, 0)$, and $T_{l}(i \kappa)$ are strictly positive, and hence each of the four $A_{j}(\kappa)$ is strictly positive.

Let

$$
u(x ; \kappa):= \begin{cases}e^{-\lambda x} v(i \kappa, x), & x \geq 0, \\ e^{\kappa x} v(i \kappa, x), & x \leq 0,\end{cases}
$$

where $\lambda$ is the constant defined in terms of $\kappa$ as

$$
\lambda=\sqrt{\kappa^{2}+c^{2}}
$$

and $c$ is the constant appearing in (1.2). Even though $v(i \kappa, x)$ is unbounded as $x \rightarrow \pm \infty$, we will see that $u(x ; \kappa)$ has nicer properties that will be useful later on. 
Proposition 3.2 Assume that $V$ satisfies (1.2) for some $c \geq 0$ and that $\kappa>\kappa_{N}$ (if there are no bound states, let $\kappa>0$ ). Then:

(i) $u(x ; \kappa)$ and $u^{\prime}(x ; \kappa)$ are continuous and bounded in $x \in \mathbf{R}$.

(ii) The spatial asymptotics of $u(x ; \kappa)$ and $u^{\prime}(x ; \kappa)$ are given by

$$
\begin{gathered}
u^{\prime}(x ; \kappa)=o(1 / x), \quad x \rightarrow \pm \infty, \\
u(x ; \kappa)=\left\{\begin{array}{l}
\frac{f_{l}(i \kappa, 0)}{2 \lambda}+o(1), \quad x \rightarrow+\infty, \\
-\frac{f_{r}(i \kappa, 0)}{2 \kappa}+o(1), \quad x \rightarrow-\infty .
\end{array}\right.
\end{gathered}
$$

PROOF: Using (3.1) and (3.9) in $(1.1)$ we see that $u(\cdot ; \kappa)$ and $u^{\prime}(\cdot ; \kappa)$ are both continuous and satisfy $u(0 ; \kappa)=0$ and $u^{\prime}(0 ; \kappa)=1$. Thus, from (3.2), (3.3), and (3.9) we get

$$
\begin{gathered}
u(x ; \kappa)=\left\{\begin{array}{l}
\frac{1}{2 \lambda}\left[1-e^{-2 \lambda x}\right]+\frac{1}{2 \lambda} \int_{0}^{x} d y\left[1-e^{-2 \lambda(x-y)}\right]\left[V(y)-c^{2}\right] u(y ; \kappa), \quad x \geq 0, \\
\frac{1}{2 \kappa}\left[e^{2 \kappa x}-1\right]+\frac{1}{2 \kappa} \int_{x}^{0} d y\left[1-e^{-2 \kappa(y-x)}\right] V(y) u(y ; \kappa), \quad x \leq 0,
\end{array}\right. \\
u^{\prime}(x ; \kappa)= \begin{cases}e^{-2 \lambda x}+\int_{0}^{x} d y e^{-2 \lambda(x-y)}\left[V(y)-c^{2}\right] u(y ; \kappa), & x \geq 0, \\
e^{2 \kappa x}-\int_{x}^{0} d y e^{-2 \kappa(y-x)} V(y) u(y ; \kappa), & x \leq 0 .\end{cases}
\end{gathered}
$$

The Volterra equation (3.13) can be solved by using iteration, and we get

$$
|u(x ; \kappa)| \leq\left\{\begin{array}{l}
\frac{1}{\lambda} \exp \left(\frac{1}{\lambda} \int_{0}^{x} d y\left|V(y)-c^{2}\right|\right), \quad x \geq 0, \\
\frac{1}{\kappa} \exp \left(\frac{1}{\kappa} \int_{x}^{0} d y|V(y)|\right), \quad x \leq 0 .
\end{array}\right.
$$

Because of $(1.2)$, we see from $(3.15)$ that $u(x ; \kappa)$ is bounded in $x \in \mathbf{R}$. Letting $C$ denote a generic constant and using $|u(x ; \kappa)| \leq C$ in (3.14), we see that $u^{\prime}(x ; \kappa)$ is bounded in 
$x \in \mathbf{R}$. In fact, from (3.14) we get the following estimates. When $x>0$ we have

$$
\begin{aligned}
\left|u^{\prime}(x ; \kappa)\right| & \leq e^{-2 \lambda x}+C \int_{0}^{x / 2} d y e^{-2 \lambda(x-y)}\left|V(y)-c^{2}\right|+\frac{2 C}{x} \int_{x / 2}^{x} d y y e^{-2 \lambda(x-y)}\left|V(y)-c^{2}\right| \\
& \leq e^{-2 \lambda x}+C e^{-\lambda x} \int_{0}^{x / 2} d y\left|V(y)-c^{2}\right|+\frac{2 C}{x} \int_{x / 2}^{x} d y y e^{-2 \lambda(x-y)}\left|V(y)-c^{2}\right| .
\end{aligned}
$$

From (1.2) it follows that the last integral in (3.16) is $o(1)$ as $x \rightarrow+\infty$, and hence $u^{\prime}(x ; \kappa)=$ $o(1 / x)$ as $x \rightarrow+\infty$. Similarly, when $x<0$ we have

$$
\begin{aligned}
\left|u^{\prime}(x ; \kappa)\right| & \leq e^{2 \kappa x}+C \int_{x / 2}^{0} d y e^{-2 \kappa(y-x)}|V(y)|+\frac{2 C}{|x|} \int_{x}^{x / 2} d y|y| e^{-2 \kappa(y-x)}|V(y)| \\
& \leq e^{2 \kappa x}+C e^{\kappa x} \int_{x / 2}^{0} d y|V(y)|+\frac{2 C}{|x|} \int_{x}^{x / 2} d y|y| e^{-2 \kappa(y-x)}|V(y)|,
\end{aligned}
$$

and since the last integral in (3.17) is $o(1)$ as $x \rightarrow-\infty$, it follows that $u^{\prime}(x ; \kappa)=o(1 / x)$ as $x \rightarrow-\infty$. Thus, (3.11) has been established. Letting

$$
m_{l}(k, x):=e^{-i \gamma x} f_{l}(k, x)
$$

from (2.1) we get

$$
m_{l}(i \kappa, x)=1+o(1), \quad m_{l}^{\prime}(i \kappa, x)=o(1), \quad x \rightarrow+\infty .
$$

The first Wronskian identity in (3.4) can be written as

$$
f_{l}(i \kappa, 0)=m_{l}(i \kappa, x) u^{\prime}(x ; \kappa)+\left[2 \lambda m_{l}(i \kappa, x)-m_{l}^{\prime}(i \kappa, x)\right] u(x ; \kappa) .
$$

Letting $x \rightarrow+\infty$ in (3.19) and recalling that $f_{l}(i \kappa, 0)>0$, with the help of (3.11) and (3.19), we get (3.12) as $x \rightarrow+\infty$. Similarly, letting

$$
m_{r}(k, x):=e^{i k x} f_{r}(k, x),
$$

from (2.3) we get

$$
m_{r}(i \kappa, x)=1+o(1), \quad m_{r}^{\prime}(i \kappa, x)=o(1), \quad x \rightarrow-\infty .
$$


The second Wronskian identity in (3.4) can be written as

$$
f_{r}(i \kappa, 0)=m_{r}(i \kappa, x) u^{\prime}(x ; \kappa)-\left[2 \kappa m_{r}(i \kappa, x)+m_{r}^{\prime}(i \kappa, x)\right] u(x ; \kappa)
$$

Letting $x \rightarrow-\infty$ in (3.22) and recalling that $f_{r}(i \kappa, 0)>0$, using (3.11) and (3.21), we establish (3.12) as $x \rightarrow-\infty$.

Proposition 3.3 Assume that $V$ satisfies (1.2) for some $c \geq 0$ and that $\kappa>\kappa_{N}$ (if there are no bound states, let $\kappa>0)$. Then $u^{\prime}(\cdot ; \kappa)$ belongs to $L_{1}^{1}(\mathbf{R})$, where $u(x ; \kappa)$ is the quantity defined in (3.9).

PROOF: As shown in Proposition 3.2(i), $u^{\prime}(\cdot ; \kappa)$ is continuous. Thus, as seen from (3.16) and (3.17), in order to prove that $u^{\prime}(\cdot ; \kappa)$ belongs to $L_{1}^{1}(\mathbf{R})$, it is enough to prove that $I_{1}$ and $I_{2}$ are finite, where we have defined

$$
\begin{aligned}
& I_{1}:=\int_{2 a}^{\infty} d x\left(1+\frac{1}{x}\right) \int_{x / 2}^{x} d y y e^{-2 \lambda(x-y)}\left|V(y)-c^{2}\right|, \\
& I_{2}:=\int_{-\infty}^{-2 a} d x\left(1+\frac{1}{|x|}\right) \int_{x}^{x / 2} d y|y| e^{-2 \kappa(y-x)}|V(y)|,
\end{aligned}
$$

for some positive constant $a \geq 1$. Changing the order of integration in (3.23), we get

$$
I_{1} \leq 2 \int_{a}^{\infty} d y y e^{2 \lambda y}\left|V(y)-c^{2}\right| \int_{y}^{2 y} d x e^{-2 \lambda x}=\frac{1}{\lambda} \int_{a}^{\infty} d y y\left[1-e^{-2 \lambda y}\right]\left|V(y)-c^{2}\right|,
$$

and hence, because of (1.2), $I_{1}$ is finite. Similarly, a change of order of integration in (3.24) gives us

$$
I_{2} \leq 2 \int_{-\infty}^{-a} d y|y| e^{-2 \kappa y}|V(y)| \int_{2 y}^{y} d x e^{2 \kappa x}=\frac{1}{\kappa} \int_{-\infty}^{-a} d y|y|\left[1-e^{2 \kappa y}\right]|V(y)|
$$

and hence $I_{2}$ is also finite because of (1.2). Thus, the proof is completed.

For $\alpha>0$ let us define

$$
h(x ; \kappa, \alpha):=f_{l}(i \kappa, x)+\alpha f_{r}(i \kappa, x), \quad x \in \mathbf{R},
$$




$$
\xi(x ; \kappa, \alpha):=\frac{h^{\prime}(x ; \kappa, \alpha)}{h(x ; \kappa, \alpha)}, \quad x \in \mathbf{R} .
$$

Proposition 3.4 Assume $V$ satisfies (1.2) for some $c \geq 0$, and let $\alpha>0$ and $\kappa>\kappa_{N}$ (if there are no bound states, let $\kappa>0$ ). Then:

(i) $\xi(x ; \kappa, \alpha)$ is bounded and continuous in $x \in \mathbf{R}$.

(ii) $\xi(\cdot ; \kappa, \alpha)-\lambda$ belongs to $L_{1}^{1}\left(\mathbf{R}^{+}\right)$and $\xi(\cdot ; \kappa, \alpha)+\kappa$ belongs to $L_{1}^{1}\left(\mathbf{R}^{-}\right)$.

(iii) $\xi^{\prime}(\cdot ; \kappa, \alpha)$ exists a.e. and belongs to $L_{1}^{1}(\mathbf{R})$.

PROOF: Because of Proposition 2.1, both $h(x ; \kappa, \alpha)$ and $h^{\prime}(x ; \kappa, \alpha)$ are continuous in $x \in \mathbf{R}$. From Proposition 2.2 , it follows that $h(x ; \kappa, \alpha)$ is strictly positive, and hence $\xi(x ; \kappa, \alpha)$ is continuous in $x \in \mathbf{R}$. Using (3.5) and (3.6) in (3.25), with the help of (3.7)(3.10), we obtain

$$
h(x ; \kappa, \alpha)=\left\{\begin{array}{lc}
{\left[1+\alpha A_{1}(\kappa)\right] f_{l}(i \kappa, x)+\alpha A_{2}(\kappa) e^{\lambda x} u(x ; \kappa),} & x \geq 0, \\
{\left[\alpha+A_{3}(\kappa)\right] f_{r}(i \kappa, x)+A_{4}(\kappa) e^{-\kappa x} u(x ; \kappa),} & x \leq 0,
\end{array}\right.
$$

$$
h^{\prime}(x ; \kappa, \alpha)= \begin{cases}{\left[1+\alpha A_{1}(\kappa)\right] f_{l}^{\prime}(i \kappa, x)+\alpha A_{2}(\kappa) e^{\lambda x}\left[\lambda u(x ; \kappa)+u^{\prime}(x ; \kappa)\right],} & x \geq 0, \\ {\left[\alpha+A_{3}(\kappa)\right] f_{r}^{\prime}(i \kappa, x)+A_{4}(\kappa) e^{-\kappa x}\left[-\kappa u(x ; \kappa)+u^{\prime}(x ; \kappa)\right],} & x \leq 0 .\end{cases}
$$

Using (2.1), (2.3), (3.11), and (3.12) in (3.27) and (3.28), we obtain

$$
\xi(x ; \kappa, \alpha)= \begin{cases}\lambda+\frac{u^{\prime}(x ; \kappa)}{u(x ; \kappa)}+\frac{1}{u(x ; \kappa)} O\left(e^{-2 \lambda x}\right), & x \rightarrow+\infty, \\ -\kappa+\frac{u^{\prime}(x ; \kappa)}{u(x ; \kappa)}+\frac{1}{u(x ; \kappa)} O\left(e^{2 \kappa x}\right), & x \rightarrow-\infty .\end{cases}
$$

As seen from (3.12), $u(x ; \kappa)$ is bounded and remains bounded away from zero as $x \rightarrow \pm \infty$. Thus, from (3.12) and (3.29) we get

$$
\xi(x ; \kappa, \alpha)= \begin{cases}\lambda+\frac{2 \lambda u^{\prime}(x ; \kappa)}{f_{l}(i \kappa, 0)}[1+o(1)]+O\left(e^{-2 \lambda x}\right), & x \rightarrow+\infty, \\ -\kappa-\frac{2 \kappa u^{\prime}(x ; \kappa)}{f_{r}(i \kappa, 0)}[1+o(1)]+O\left(e^{2 \kappa x}\right), & x \rightarrow-\infty .\end{cases}
$$


Using (3.11) and Proposition 2.2 in (3.30), we see that $\xi(x ; \kappa, \alpha)$ is bounded for all $x \in \mathbf{R}$. Since $\xi(\cdot ; \kappa, \alpha)$ is continuous, the $L_{1}^{1}$-properties stated in (ii) follows from (3.30) and the $L_{1}^{1}$-property of $u^{\prime}(x ; \kappa)$ established in Proposition 3.3. From (1.1) and (3.26) we get

$$
\xi^{\prime}(x ; \kappa, \alpha)=V(x)+\kappa^{2}-\xi(x ; \kappa, \alpha)^{2}, \quad x \in \mathbf{R} .
$$

Using (3.10) we can write (3.31) also as

$$
\xi^{\prime}(x ; \kappa, \alpha)=V(x)-c^{2}+\lambda^{2}-\xi(x ; \kappa, \alpha)^{2}, \quad x \in \mathbf{R} .
$$

Thus, because of (1.2), as seen from (3.31) and (3.32), in order to show that $\xi^{\prime}(\cdot ; \kappa, \alpha)$ belongs to $L_{1}^{1}(\mathbf{R})$, it is sufficient to show that $\xi(\cdot ; \kappa, \alpha)^{2}-\lambda^{2}$ belongs to $L_{1}^{1}\left(\mathbf{R}^{+}\right)$and $\xi(\cdot ; \kappa, \alpha)^{2}-\kappa^{2}$ belongs to $L_{1}^{1}\left(\mathbf{R}^{-}\right)$. However, these directly follow from (i) and (ii), as seen by writing

$$
\begin{aligned}
& \xi(x ; \kappa, \alpha)^{2}-\lambda^{2}=[\xi(x ; \kappa, \alpha)-\lambda][\xi(x ; \kappa, \alpha)+\lambda], \\
& \xi(x ; \kappa, \alpha)^{2}-\kappa^{2}=[\xi(x ; \kappa, \alpha)+\kappa][\xi(x ; \kappa, \alpha)-\kappa],
\end{aligned}
$$

and using (ii) and the boundedness of $\xi(x ; \kappa, \alpha)+\lambda$ and $\xi(x ; \kappa, \alpha)-\kappa$.

\section{DARBOUX TRANSFORMATION}

Let us use a tilde to denote the quantities associated with the resulting Schrödinger equation when a bound state is added to (1.1) at $k=i \kappa$ with $\kappa>\kappa_{N}$ (with $\kappa>0$ if (1.1) has no bound states). That is, $\tilde{V}$ is the resulting potential, $\tilde{f}_{l}$ and $\tilde{f}_{r}$ are the Jost solutions, $\tilde{T}_{l}$ and $\tilde{T}_{r}$ are the transmission coefficients, and $\tilde{L}$ and $\tilde{R}$ are the reflection coefficients, from the left and from the right, respectively. We have the following result.

Theorem 4.1 Assume $V$ satisfies (1.2) for some $c \geq 0$. If a bound state is added to (1.1) at $k=i \kappa$ with $\kappa>\kappa_{N}$ (with $\kappa>0$ if (1.1) has no bound states), then

$$
\tilde{V}(x ; \kappa, \alpha)=V(x)-2 \xi^{\prime}(x ; \kappa, \alpha),
$$




$$
\begin{gathered}
\tilde{f}_{l}(k, x ; \kappa, \alpha)=\frac{1}{i(\gamma+i \lambda)}\left[f_{l}^{\prime}(k, x)-\xi(x ; \kappa, \alpha) f_{l}(k, x)\right], \\
\tilde{f}_{r}(k, x ; \kappa, \alpha)=\frac{i}{(k+i \kappa)}\left[f_{r}^{\prime}(k, x)-\xi(x ; \kappa, \alpha) f_{r}(k, x)\right], \\
\tilde{T}_{l}(k ; \kappa, \alpha)=\frac{\gamma+i \lambda}{k-i \kappa} T_{l}(k), \quad \tilde{L}(k ; \kappa, \alpha)=-\frac{k+i \kappa}{k-i \kappa} L(k), \\
\tilde{T}_{r}(k ; \kappa, \alpha)=\frac{\gamma+i \lambda}{k-i \kappa} T_{r}(k), \quad \tilde{R}(k ; \kappa, \alpha)=-\frac{\gamma+i \lambda}{\gamma-i \lambda} R(k),
\end{gathered}
$$

where $\gamma$ is as in (1.3), $\lambda$ is the constant in $(3.10)$, and $\xi(x ; \kappa, \alpha)$ is the function defined in $(3.26)$.

PROOF: It can be verified directly that $\tilde{f}_{l}$ and $\tilde{f}_{r}$ given in (4.2) and (4.3), respectively, satisfy (1.1) when the potential $V$ is replaced by $\tilde{V}$. Moreover, from the asymptotics as $x \rightarrow+\infty$ stated in (2.1) and (3.30), it follows that $\tilde{f}_{l}$ is the Jost solution from the left associated with $\tilde{V}$. Similarly, from the asymptotics as $x \rightarrow-\infty$ stated in (2.3) and (3.30), it follows that $\tilde{f}_{r}$ is the Jost solution from the right for $\tilde{V}$. With the help of (2.5), (2.6), (3.11), and (3.30), we obtain $\tilde{T}_{l}$ and $\tilde{L}$ given in (4.4). Finally, by using (2.7), (2.8), and (4.4), we establish (4.5).

Proposition 4.2 Assume $V$ satisfies (1.2) for some $c \geq 0$. If a bound state is added to (1.1) at $k=i \kappa$ with $\kappa>\kappa_{N}$ (with $\kappa>0$ if (1.1) has no bound states), then $\tilde{V}$ belongs to the same class as $V$, namely

$$
\tilde{V} \in L_{1}^{1}\left(\mathbf{R}^{-}\right), \quad \tilde{V}-c^{2} \in L_{1}^{1}\left(\mathbf{R}^{+}\right) .
$$

Moreover, the positive constant $\alpha$ introduced in (3.25) is related to the ratio of the Jost solutions of $\tilde{V}$ at the bound state $k=i \kappa$ as

$$
\frac{\tilde{f}_{l}(i \kappa, x ; \kappa, \alpha)}{\tilde{f}_{r}(i \kappa, x ; \kappa, \alpha)}=\frac{\alpha \kappa}{\lambda}
$$


where $\lambda$ is the quantity defined in (3.10). Furthermore, $\tilde{f}_{l}(i \kappa, x ; \kappa, \alpha)$ and $\tilde{f}_{r}(i \kappa, x ; \kappa, \alpha)$ are both strictly positive for all $x \in \mathbf{R}$ and decay exponentially to zero as $x \rightarrow \pm \infty$ with the asymptotics given by

$$
\begin{gathered}
\tilde{f}_{l}(i \kappa, x ; \kappa, \alpha)= \begin{cases}e^{-\lambda x}[1+o(1)], & x \rightarrow+\infty, \\
\frac{\alpha \kappa}{\lambda} e^{\kappa x}[1+o(1)], & x \rightarrow-\infty,\end{cases} \\
\tilde{f}_{r}(i \kappa, x ; \kappa, \alpha)= \begin{cases}\frac{\lambda}{\alpha \kappa} e^{-\lambda x}[1+o(1)], & x \rightarrow+\infty, \\
e^{\kappa x}[1+o(1)], & x \rightarrow-\infty .\end{cases}
\end{gathered}
$$

PROOF: We get (4.6) by using (1.2), (4.1), and Proposition 3.4(iii). Evaluating (4.2) and (4.3) at $k=i \kappa$ and using (3.26), we obtain (4.7). Since $\tilde{f}_{l}$ is asymptotic to $e^{i \gamma x}$ as $x \rightarrow+\infty$ as in (2.1) and $\tilde{f}_{r}$ to $e^{-i k x}$ as $x \rightarrow-\infty$ as in (2.3), using (4.7) we obtain (4.8) and (4.9).

In the next theorem we present the Darboux transformation when we remove from (1.1) the bound state of the lowest energy. The proof is omitted because the technique used in the proof is similar to that used in Theorem 4.1 and Proposition 4.2.

Theorem 4.3 Assume that $\tilde{V}$ satisfies (4.6) for some $c \geq 0$ and that its lowest bound-state energy corresponds to $k=i \kappa$ for some $\kappa>0$. Let $\tilde{f}_{l}(k, x)$ and $\tilde{f}_{r}(k, x)$ denote the Jost solutions for $\tilde{V}$, from the left and from the right, respectively. After the removal of the bound state at $k=i \kappa$, let us denote the resulting potential by $V$ with the corresponding Jost solutions $f_{l}(k, x)$ and $f_{r}(k, x)$. Then:

$$
\begin{gathered}
V(x)=\tilde{V}(x)-2 \eta^{\prime}(x), \\
f_{l}(k, x)=\frac{1}{i(\gamma-i \lambda)}\left[\tilde{f}_{l}^{\prime}(k, x)-\eta(x) \tilde{f}_{l}(k, x)\right], \\
f_{r}(k, x)=\frac{i}{(k-i \kappa)}\left[\tilde{f}_{r}^{\prime}(k, x)-\eta(x) \tilde{f}_{r}(k, x)\right],
\end{gathered}
$$

where $\gamma$ is as in (1.3), $\lambda$ is as in (3.10), and $\eta(x):=\tilde{f}_{l}^{\prime}(i \kappa, x) / \tilde{f}_{l}(i \kappa, x)$. Moreover, $\eta(+\infty)=$ $-\lambda, \eta(-\infty)=\kappa, \eta^{\prime} \in L_{1}^{1}(\mathbf{R})$, and $V$ belongs to the same class as $\tilde{V}$ specified in (4.6). 
Using Propositions 4.1 and 4.2 in a recursive manner, we obtain the following result.

Corollary 4.4 Assume that $V$ satisfies (1.2) for some $c \geq 0$ and it has bound states at $k=i \kappa_{j}$ for $j=1, \ldots, N ;$ let $\lambda_{j}=\sqrt{\kappa_{j}^{2}+c^{2}}$. Then:

$$
\begin{gathered}
T_{l}(k)=T_{l}^{[0]}(k) \prod_{j=1}^{N} \frac{\gamma+i \lambda_{j}}{k-i \kappa_{j}}, \quad T_{r}(k)=T_{r}^{[0]}(k) \prod_{j=1}^{N} \frac{\gamma+i \lambda_{j}}{k-i \kappa_{j}}, \\
L(k)=(-1)^{N} L^{[0]}(k) \prod_{j=1}^{N} \frac{k+i \kappa_{j}}{k-i \kappa_{j}}, \quad R(k)=(-1)^{N} R^{[0]}(k) \prod_{j=1}^{N} \frac{\gamma+i \lambda_{j}}{\gamma-i \lambda_{j}},
\end{gathered}
$$

where $T_{l}^{[0]}, T_{r}^{[0]}, L^{[0]}$, and $R^{[0]}$ are the scattering coefficients corresponding to the potential $V^{[0]}$ obtained from $V$ by removing all its bound states, and $V^{[0]}$ belongs to the same class as $V$ does, i.e. $V^{[0]} \in L_{1}^{1}\left(\mathbf{R}^{-}\right)$and $V^{[0]}-c^{2} \in L_{1}^{1}\left(\mathbf{R}^{+}\right)$.

Notice that if we let $c \rightarrow 0$ in (4.2)-(4.5), (4.10), and (4.11), then we obtain the well-known Darboux transformations $[1,2]$ for the standard Schrödinger equation.

In certain applications [11-14] in materials science, the potential $V(x)$ has support in $\mathbf{R}^{+}$. In such cases, we show in the next proposition that the constant $\alpha$ appearing in (3.25) must be chosen in a unique manner in order not to change the potential for $x<0$.

Proposition 4.5 Assume that $V$ satisfies (1.2) for some $c \geq 0$, vanishes for $x<0$, and has bound states at $k=i \kappa_{j}$ for $j=1, \ldots, N$. If a bound state is added to $V$ at $k=i \kappa$ with $\kappa>\kappa_{N}$ (with $\kappa>0$ if (1.1) has no bound states), then $\tilde{V}$ also vanishes for $x<0$ if and only if the constant $\alpha$ appearing in (3.25) is chosen as

$$
\alpha=-\frac{L(i \kappa)}{T_{l}(i \kappa)} .
$$

PROOF: When $V$ vanishes for $x<0$, its Jost solutions on $\mathbf{R}^{-}$are determined by the scattering coefficients as

$$
f_{l}(k, x)=\frac{e^{i k x}+L(k) e^{-i k x}}{T_{l}(k)}, \quad f_{r}(k, x)=e^{-i k x}, \quad x \leq 0 .
$$


Using (3.25) and (4.13) in (3.26), we get

$$
\xi(x ; \kappa, \alpha)=-\kappa \frac{e^{-\kappa x} / T_{l}(i \kappa)-\left[\alpha+L(i \kappa) / T_{l}(i \kappa)\right] e^{\kappa x}}{e^{-\kappa x} / T_{l}(i \kappa)+\left[\alpha+L(i \kappa) / T_{l}(i \kappa)\right] e^{\kappa x}}, \quad x \leq 0
$$

and hence, because of (4.1), $\tilde{V}$ vanishes for $x<0$, i.e. $\xi(x ; \kappa, \alpha)$ is a constant, if and only if (4.12) is satisfied.

\section{REPRESENTATIONS FOR SCATTERING COEFFICIENTS}

The integral relation $(2.2)$ is not suitable to obtain the asymptotics of $f_{l}(k, x)$ as $x \rightarrow-\infty$. For this we can use the representation

$$
2 i k f_{l}(k, x)=B_{1}(k) e^{i k x}+B_{2}(k) e^{-i k x}+\int_{x}^{0} d y\left[e^{i k(y-x)}-e^{-i k(y-x)}\right] V(y) f_{l}(k, y)
$$

where we have defined

$$
B_{1}(k):=i k f_{l}(k, 0)+f_{l}^{\prime}(k, 0), \quad B_{2}(k):=i k f_{l}(k, 0)-f_{l}^{\prime}(k, 0) .
$$

It can be easily checked that $f_{l}(k, x)$ given in (5.1) satisfies (1.1) and the appropriate boundary conditions at $x=0$. Letting

$$
p_{l}(k, x):=e^{-i k x} f_{l}(k, x)
$$

we can write $(5.1)$ as

$$
2 i k p_{l}(k, x)=B_{1}(k)+B_{2}(k) e^{-2 i k x}+\int_{x}^{0} d y\left[e^{2 i k(y-x)}-1\right] V(y) p_{l}(k, y) .
$$

By iterating (5.4), for $x \leq 0$ we get

$$
\left|p_{l}(k, x)\right| \leq \frac{1}{2|k|}\left[\left|B_{1}(k)\right|+\left|B_{2}(k)\right|\right] \exp \left(\frac{1}{|k|} \int_{-\infty}^{0} d y|V(y)|\right), \quad k \in \overline{\mathbf{C}^{+}} \backslash\{0\}
$$

With the help of $(2.5),(5.1),(5.2),(5.4)$, and (5.5), we obtain

$$
\frac{2 i k}{T_{l}(k)}=i k f_{l}(k, 0)+f_{l}^{\prime}(k, 0)-\int_{-\infty}^{0} d y V(y) p_{l}(k, y), \quad k \in \overline{\mathbf{C}^{+}} \backslash\{0\},
$$




$$
\frac{2 i k L(k)}{T_{l}(k)}=i k f_{l}(k, 0)-f_{l}^{\prime}(k, 0)+\int_{-\infty}^{0} d y e^{2 i k y} V(y) p_{l}(k, y), \quad k \in \mathbf{R} \backslash\{0\} .
$$

For each fixed $k \in \mathbf{C}^{+}$, letting $x \rightarrow-\infty$ in (5.4) and using (5.6), we get

$$
2 i k p_{l}(k, x)=B_{1}(k)-\int_{-\infty}^{0} d y V(y) p_{l}(k, y)+o(1)=\frac{2 i k}{T_{l}(k)}+o(1)
$$

and hence from (5.2) and (5.8) we have

$$
e^{-i k x} f_{l}(k, x)=\frac{1}{T_{l}(k)}[1+o(1)], \quad k \in \mathbf{C}^{+}, \quad x \rightarrow-\infty .
$$

In the integrand in (5.7), when $k \in \mathbf{C}^{+}$, the factor $e^{2 i k y}$ grows exponentially as $y \rightarrow-\infty$; hence, unless $V(y)$ decays faster, the integral does not converge and thus $L(k)$ does not have an extension from real $k$ values to complex ones.

In a similar manner, in order to study the asymptotics of $f_{r}(k, x)$ as $x \rightarrow+\infty$, instead of (2.4) we will use the integral relation

$$
2 i \gamma f_{r}(k, x)=B_{3}(k) e^{i \gamma x}+B_{4}(k) e^{-i \gamma x}+\int_{0}^{x} d y\left[e^{i \gamma(x-y)}-e^{-i \gamma(x-y)}\right]\left[V(y)-c^{2}\right] f_{r}(k, y),
$$

where we have defined

$$
B_{3}(k):=i \gamma f_{r}(k, 0)+f_{r}^{\prime}(k, 0), \quad B_{4}(k):=i \gamma f_{r}(k, 0)-f_{r}^{\prime}(k, 0) .
$$

It can be checked that $f_{r}(k, x)$ given in (5.9) satisfies (1.1) and the appropriate boundary conditions at $x=0$. Letting

$$
p_{r}(k, x):=e^{i \gamma x} f_{r}(k, x),
$$

we can write $(5.9)$ as

$$
2 i \gamma p_{r}(k, x)=B_{3}(k) e^{2 i \gamma x}+B_{4}(k)+\int_{0}^{x} d y\left[e^{2 i \gamma(x-y)}-1\right]\left[V(y)-c^{2}\right] p_{r}(k, y) .
$$


Iterating (5.12), for $x \geq 0$ we get

$$
\left|p_{r}(k, x)\right| \leq \frac{1}{2|\gamma|}\left[\left|B_{3}(k)\right|+\left|B_{4}(k)\right|\right] \exp \left(\frac{1}{|\gamma|} \int_{0}^{\infty} d y\left|V(y)-c^{2}\right|\right), \quad \gamma \in \overline{\mathbf{C}^{+}} \backslash\{0\} .
$$

Using (2.6), (5.9), (5.10), (5.12), and (5.13), we obtain

$$
\begin{gathered}
\frac{2 i \gamma}{T_{r}(k)}=i \gamma f_{r}(k, 0)-f_{r}^{\prime}(k, 0)-\int_{0}^{\infty} d y\left[V(y)-c^{2}\right] p_{r}(k, y), \quad \gamma \in \overline{\mathbf{C}^{+}} \backslash\{0\} \\
\frac{2 i \gamma R(k)}{T_{r}(k)}=i \gamma f_{r}(k, 0)+f_{r}^{\prime}(k, 0)+\int_{0}^{\infty} d y e^{-2 i \gamma y}\left[V(y)-c^{2}\right] p_{r}(k, y), \quad \gamma \in \mathbf{R} \backslash\{0\}
\end{gathered}
$$

Using (2.7), (2.8), (5.6), and (5.7), we can extend $R(k)$ and $T_{r}(k)$ to $k \in[-c, c]$ as well. In (5.12), for each fixed $\gamma \in \mathbf{C}^{+}$, letting $x \rightarrow+\infty$ we get

$$
2 i \gamma p_{r}(k, x)=B_{4}(k)-\int_{0}^{\infty} d y\left[V(y)-c^{2}\right] p_{r}(k, y)+o(1)=\frac{2 i \gamma}{T_{r}(k)}+o(1),
$$

and hence from (5.11) and (5.16) we get

$$
e^{i \gamma x} f_{r}(k, x)=\frac{1}{T_{r}(k)}[1+o(1)], \quad \gamma \in \mathbf{C}^{+}, \quad x \rightarrow+\infty .
$$

In the integrand in (5.15), when $\gamma \in \mathbf{C}^{+}$, the factor $e^{-2 i \gamma y}$ grows exponentially as $y \rightarrow+\infty$, and hence unless $V(y)-c^{2}$ decays faster, the integral does not converge and $R(k)$ does not have an extension from real $k$ values to complex ones.

Proposition 5.1 Assume $V$ satisfies (1.2) for some $c \geq 0$. Then, $p_{l}(k, x)$ and $p_{r}(k, x)$ defined in (5.3) and (5.11), respectively, have the following properties:

(i) For each $x \in \mathbf{R}, p_{l}(\cdot, x)$ and $p_{r}(\cdot, x)$ are analytic in $k \in \mathbf{C}^{+}$and continuous in $k \in \overline{\mathbf{C}^{+}}$.

(ii) Uniformly in $x \in \mathbf{R}^{-}$we have

$$
p_{l}(k, x)=1+O(1 / k), \quad p_{l}^{\prime}(k, x)=o(1), \quad k \rightarrow \infty \text { in } \overline{\mathbf{C}^{+}} .
$$


(iii) Uniformly in $x \in \mathbf{R}^{+}$we have

$$
p_{r}(k, x)=1+O(1 / k), \quad p_{r}^{\prime}(k, x)=o(1), \quad k \rightarrow \infty \text { in } \overline{\mathbf{C}^{+}} .
$$

PROOF: Because of (5.3) and (5.11), the analyticity and continuity properties stated in (i) directly follow from Proposition 2.1. From (2.2) and (5.3), by proceeding $[1,2]$ as in the standard Schrödinger equation with $c=0$, we get

$$
m_{l}(k, 0)=1+O(1 / \gamma), \quad m_{l}^{\prime}(k, 0)=o(1), \quad \gamma \rightarrow \infty \text { in } \overline{\mathbf{C}^{+}}
$$

and from (1.3) we have $\gamma=k+O(1 / k)$ as $k \rightarrow \infty$ in $\overline{\mathbf{C}^{+}}$. Thus, with the help of (3.18), from (5.2) we obtain

$$
\begin{aligned}
& B_{1}(k)=i(k+\gamma)[1+O(1 / \gamma)]+o(1)=2 i k+o(1), \quad k \rightarrow \infty \text { in } \overline{\mathbf{C}^{+}}, \\
& B_{2}(k)=i(k-\gamma)[1+O(1 / \gamma)]+o(1)=o(1), \quad k \rightarrow \infty \text { in } \overline{\mathbf{C}^{+}} .
\end{aligned}
$$

Note that $\left|e^{-2 i k x}\right| \leq 1$ when $x \in \mathbf{R}^{-}$and $k \in \overline{\mathbf{C}^{+}}$. Using iteration on (5.4), we find that

$$
p_{l}(k, x)-\frac{1}{2 i k}\left[B_{1}(k)+B_{2}(k) e^{-2 i k x}\right]=O(1 / k), \quad k \rightarrow \infty \text { in } \overline{\mathbf{C}^{+}} .
$$

Thus, from (5.17)-(5.19) we obtain $p_{l}(k, x)=1+O(1 / k)$ as $k \rightarrow \infty$ in $\overline{\mathbf{C}^{+}}$uniformly for all $x \in \mathbf{R}^{-}$. From (5.4) we obtain

$$
p_{l}^{\prime}(k, x)=-B_{2}(k)-\int_{x}^{0} d y e^{2 i k(y-x)} V(y) p_{l}(k, y) .
$$

Iterating (5.20) and using (5.18) and (5.19), we get $p_{l}^{\prime}(k, x)=o(1)$ as $k \rightarrow \infty$ in $\overline{\mathbf{C}^{+}}$ uniformly for all $x \in \mathbf{R}^{-}$. Thus, the proof of (ii) is complete. The proof of (iii) is similar to that of (ii), and it is obtained by using (2.4), (3.20), (5.11), and (5.12).

The integral representations (5.6), (5.7), (5.14), and (5.15) can be used to establish various properties of the scattering coefficients such as their small- $k$ and large- $k$ asymptotics. 
For example, their large- $k$ asymptotics can be obtained with the help of Proposition 5.1. However, such derivations will not be given in this paper, and we let the interested reader to extract such properties from those integral representations.

Acknowledgments. The author has benefited from some discussions with Jorge Zubelli and Fritz Gesztesy. The research leading to this article was supported in part by the National Science Foundation under grant DMS-9803219.

\section{REFERENCES}

[1] P. Deift and E. Trubowitz, Inverse scattering on the line, Comm. Pure Appl. Math. 32 (1979), 121-251.

[2] K. Chadan and P. C. Sabatier, "Inverse Problems in Quantum Scattering Theory," 2nd ed., Springer, New York, 1989.

[3] F. Gesztesy, B. Simon, and G. Teschl, Spectral deformations of one-dimensional Schrödinger operators, J. Anal. Math. 70 (1996), 267-324.

[4] V. Buslaev and V. Fomin, An inverse scattering problem for the one-dimensional Schrödinger equation on the entire axis, Vestnik Leningrad. Univ. 17 (1962), 56-64 (Russian).

[5] A. Cohen and T. Kappeler, Scattering and inverse scattering for steplike potentials in the Schrödinger equation, Indiana Univ. Math. J. 34 (1985), 127-180.

[6] J. Legendre, "Problème Inverse de Schrödinger sur la Ligne avec Conditions Dissymmétriques et Applications," Ph.D. thesis, Acad. Montpellier, Univ. Sci. Tech. Languedoc, 1982.

[7] T. Aktosun, On the Schrödinger equation with steplike potentials, J. Math. Phys., 
to appear (1999) [the manuscript is available from the author's home page at the url http://www.math.ndsu.nodak.edu/faculty/aktosun/preprints.html].

[8] E. B. Davies and B. Simon, Scattering theory for systems with different spatial asymptotics on the left and right, Comm. Math. Phys. 63 (1978), 277-301.

[9] F. Gesztesy, R. Nowell, and W. Pötz, One-dimensional scattering theory for quantum systems with nontrivial spatial asymptotics, Diff. Integral Eqs. 10 (1997), 521-546.

[10] X. L. Zhou and S. H. Chen, Theoretical foundations of X-ray and neutron reflectometry, Phys. Rep. 257 (1995), 223-348.

[11] G. Felcher and T. Russell (eds.), Proceedings of the workshop on methods of analysis and interpretation of neutron reflectivity data, Phys. B 173 (1991).

[12] G. Felcher and H. You (eds.), Proceedings of the 4th international conference on surface X-ray and neutron scattering, Phys. B 221 (1996).

[13] C. F. Majkrzak and N. F. Berk, Exact determination of the phase in neutron reflectometry, Phys. Rev. B 52 (1995), 10827-10830.

[14] V. O. de Haan, A. A. van Well, P. E. Sacks, S. Adenwalla, and G. P. Felcher, Toward the solution of the inverse problem in neutron reflectometry, Phys. B 221 (1996), $524-532$.

[15] T. Aktosun and P. Sacks, Phase recovery with nondecaying potentials, in preparation (1999).

[16] T. Aktosun, M. Klaus, and C. van der Mee, Wave scattering with absorption, J. Math. Phys. 39 (1998), 1957-1992. 